Conference Proceedings Paper

\title{
Using Olive Stone Powder for Biodegradation of Bio- based Polyamide 5.6
}

\author{
Şebnem Gülel 1,* and Yüksel Güvenilir ${ }^{2}$ \\ Published: 4 November 2020 \\ 1 Istanbul Technical University, Polymer Science and Technology, 34469, Istanbul, Turkey; \\ sebnemgulel@gmail.com \\ 2 Istanbul Technical University, Department of Chemical Engineering, 34469, Istanbul, Turkey; \\ avcibasi@itu.edu.tr \\ * Correspondence: sebnemgulel@gmail.com; Tel.:+90-530-141-06-24
}

\begin{abstract}
Polymers are extensively used advanced materials. The most commonly used polymers in industry are non-biodegradable and petroleum derived. The increasing demand for these types of polymers brings along the problem of accumulation of plastic waste in the environment and depletion of fossil resources. At this point, biodegradability of polymers gains great importance as well as bio-based polymers produced from renewable resources. In this study, bio-based polyamide 5.6 polymer (PA56) was incorporated with olive stone powder (OSP) in order to manufacture a biodegradable polyamide compound, and its degradability was investigated. The olive stone powder was incorporated into polyamide 5.6 at $10 \%(\mathrm{w} / \mathrm{w})$ with a twin screw extruder in order to manufacture the compound, PA56/OSP10. The characterization of the PA56/ OSP10 compound was done using Fourier transform infrared (FTIR) spectroscopy. The biodegradability of the PA56/OSP10 compound was examined through a natural soil burial test which lasted for 6 months. The sign of degradation was assessed by both weight loss measurements and visual observations. At the end of 6 months, 5.24\% weight loss and surface deformation were determined for the PA56/OSP10 compound. These results suggest that olive stone powder can be considered as a green alternative to conventional biodegradation additives for polymer compounding.
\end{abstract}

Keywords: biodegradability; olive stone powder; bio-based; polyamide 5.6; compounding

\section{Introduction}

The worldwide production of plastics was 359 million tonnes in 2018 [1]. This number is expected to reach 400 million tonnes in 2020 [2]. Since most of these polymers are non-biodegradable, they do not decompose naturally and cause accumulation of plastic waste in the environment. At this point, biodegradability of polymers gains great importance.

Polyamides are the petroleum derived polymers with repeating amide linkage $(-\mathrm{C}(\mathrm{O})-\mathrm{NH}-)$ in their backbone. However, polyamide 5.6 (PA56) is classified as a bio-based polyamide since cadaverine monomer, which is one of its building blocks, is synthesized via biological method from renewable resources [3].

Biodegradability is the breakdown of organic substances by living microorganisms [4]. This process takes place via the secreted enzymes, which lead to specific chemical reactions for the cleavage of specific chemical bonds [5].

Olive stone powder (OSP) is produced by grinding olive stones, which are the soil wastes of the olive oil manufacturing industry. The reported researches in the literature about utilization of OSP in polymer industry includes its usage as a filler to polylactic acid matrix biocomposites [6], as 
The 1st International Electronic Conference on "Green" Polymer Materials 2020, 5-25 November 2020 reinforcement in polypropylene composites [7], and as a filler for polyvinyl composites [8]. However, there is not any study in the literature about the biodegradability of bio-based polyamide 5.6 compound with olive stone powder.

In this study, a novel bio-based polyamide compound was manufactured by incorporating olive stone powder with bio-based polyamide 5.6 polymer. In addition, biodegradability of this compound was investigated through a natural soil burial test for 6 months. The characterization of the compound was done with Fourier transform infrared (FTIR) spectroscopy, and the biodegradation is assessed with weight loss measurements and visual observations. The aim of the present work is to evaluate the olive stone powder as a green biodegradability additive for bio-based polyamide 5.6.

\section{Experiments}

\subsection{Materials}

Bio-based polyamide 5.6 (PA56), a 45\% renewable polyamide grade, was obtained from Cathay Industrial Biotech Ltd., China. The PA56 has a specific gravity of $1.14 \mathrm{~g} / \mathrm{cm}^{3}$ and a relative viscosity of $2.7 \mathrm{cP}$. Olive stone powder (OSP) was supplied by Biotechnology Laboratory of Istanbul Technical University, Turkey.

\subsection{Compounding and Sample Preparation}

OSP was introduced to PA56 at $10 \%(w / w)$ with a twin screw extruder, Haake MiniLab II (Thermo Scientific, Germany), in order to manufacture the biodegradable polyamide compound, PA56/OSP10. The compounding was done at $265^{\circ} \mathrm{C}$ for 10 minutes with a screw speed of $100 \mathrm{rpm}$ and test samples were prepared with injection molding. Neat polyamide 5.6 pellets were also injection molded and were used as controls during the applied tests.

\subsection{Fourier transform infrared (FTIR) analysis}

The characterization of the polyamide 5.6, olive stone powder and PA56/OSP10 were done by using FTIR spectrophotometer, Spectrum100 (Perkin Elmer, USA). The applied scan range was 4000 $-650 \mathrm{~cm}^{-1}$, the resolution was $4.0 \mathrm{~cm}^{-1}$ and the number of scans was 64 .

\subsection{Biodegradability}

The biodegradability of the PA56/OSP10 compound was examined through a natural soil burial test for 6 months. The test was conducted at the Istanbul Technical University, Istanbul (latitude: 41.105595, longitude 29.025339) from February 2020 to July 2020. Meteorological data were obtained from Turkish State Meteorological Service [9]. The used test samples were $3 \mathrm{~cm}$ by $3 \mathrm{~cm}$ square pieces, and five test samples were used for PA56/OSP10 compound. The molded neat polyamide 5.6 was used as a control. The samples were weighed after dried in an air-drying oven at $80{ }^{\circ} \mathrm{C}$ for $24 \mathrm{~h}$. Then they were buried into the soil. Every 30 days, the samples were dug out for examination. They were washed in distilled water and dried before undergoing weight loss measurements and visual observations. For visual observation, images of the compounds and controls were taken before and after the soil burial in order to spot the differences.

\section{Results and Discussion}

\subsection{Fourier transform infrared (FTIR) analysis}

The FTIR spectrum of the manufactured PA56/OSP10 compound was overlapped with the spectrum of PA56, and given in Figure 1. Both PA56 and PA56/OSP10 exhibited characteristic FTIR absorption bands of the amide linkage at $3304 \mathrm{~cm}^{-1}$ with $\mathrm{N}-\mathrm{H}$ stretching, at $1632 \mathrm{~cm}^{-1}$ with $\mathrm{C}=\mathrm{O}$ stretching and at $1533 \mathrm{~cm}^{-1}$ with $\mathrm{N}-\mathrm{H}$ bending. The main difference between their spectra was observed when PA56/OSP10 compound presented two peaks, at $1080 \mathrm{~cm}^{-1}$ and at $1018 \mathrm{~cm}^{-1}$. However, 
The 1st International Electronic Conference on "Green" Polymer Materials 2020, 5-25 November 2020 neat PA56 showed no peaks at these wavelengths. These arisen peaks are corresponding to C-O-C stretching, which indicates the presence of the OSP in the PA56/OSP10 compound. This finding is important since it proves the incorporation of OSP into the PA56, meaning the extrusion compounding process was successful.

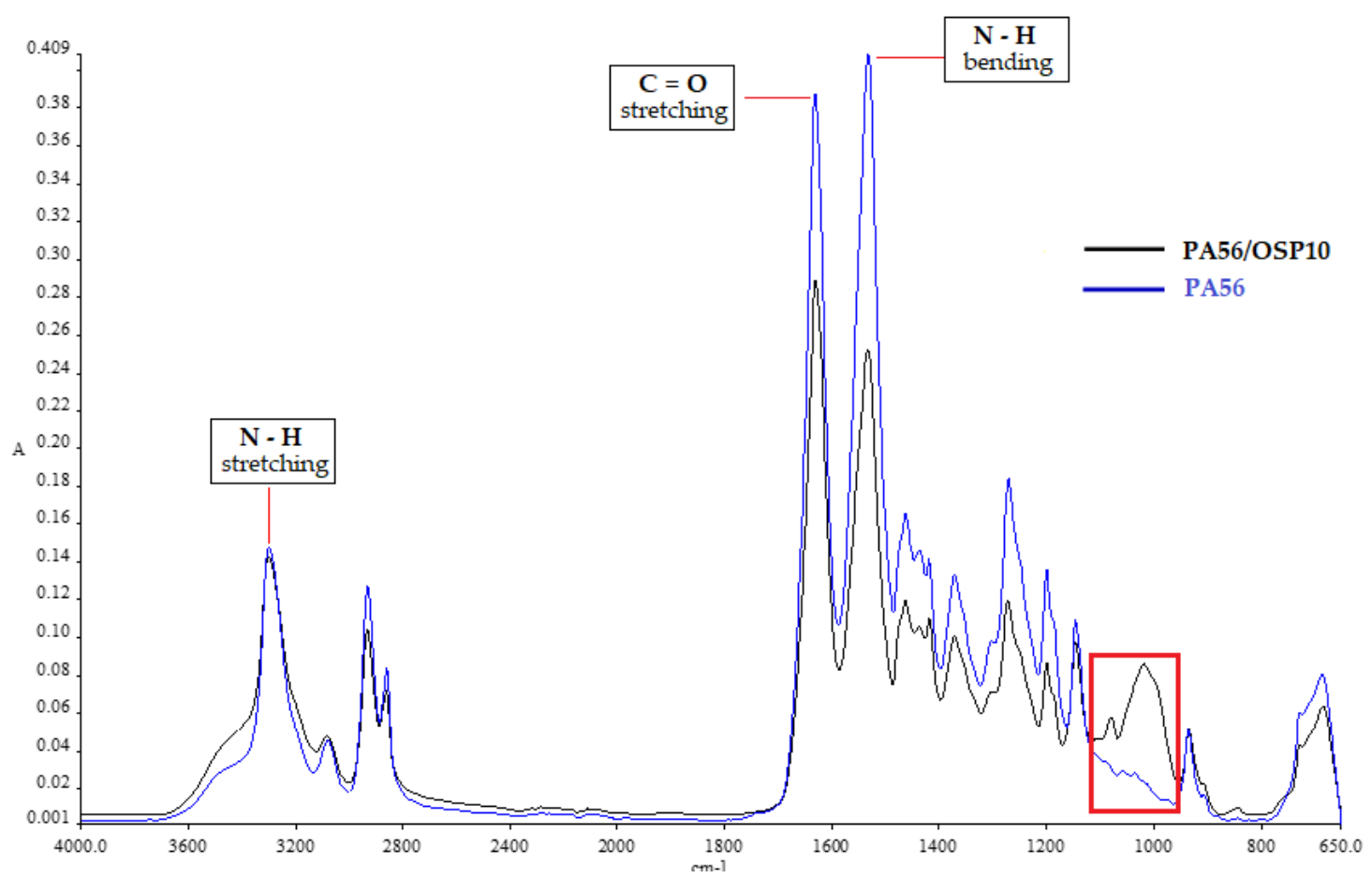

Figure 1. The FTIR spectra of the PA56/OSP10 compound and the polyamide 5.6

\subsection{Biodegradability}

Even though bio-based materials are composed of or derived from biological products, it does not mean that the material is biodegradable [10,11]. Thus, the biodegradability of bio-based polyamide 5.6 was investigated with natural soil burial test. At the end of 6 months, 5.24\% weight loss was determined for the manufactured PA56/OSP10 compound. The weight percentage of the OSP, types of microorganism present in the soil, meteorological data based on location, and the burial time of the samples in soil are the factors which affect the biodegradability. By changing any of these factors, biodegradability of the polymer compounds can also be changed.

The changes in the surface apperances of the buried and the unburied samples were done from the taken images. The buried PA56/OSP10 samples showed surface deformations, abrasions and discolorations, whereas buried PA56 samples displayed only discoloration. These findings also suggest the biodegradation of the PA56/OSP10 compound during the natural soil burial test.

\section{Conclusions}

This study investigated the biodegradability effect of olive stone powder on bio-based polyamide 5.6 polymer. For this purpose, a novel bio-based polyamide compound, PA56/OSP10, was successfully manufactured from 90\% (w/w) bio-based polyamide 5.6 and 10\% (w/w) olive stone powder, by extrusion compounding method. Natural soil burial test was applied and the buried PA56/OSP10 compound showed 5.24\% (w/w) degradation at the end of 6 months. Surface deformations, abrasions and discolorations were also observed for these compounds. In the light of these findings, olive stone powder can be considered as a green biodegradability additive for the biobased polyamide 5.6 polymer. 
The 1st International Electronic Conference on "Green" Polymer Materials 2020, 5-25 November 2020

Acknowledgments: This work was supported by Research Fund of the Istanbul Technical University. Project Number: 42182.

Author Contributions: S..G. conceived and designed the experiments; performed the experiments; analyzed the data; contributed reagents/materials/analysis tools and wrote the paper; Y.G. advised and supervised.

Conflicts of Interest: The authors declare no conflict of interest. The founding sponsors had no role in the design of the study; in the collection, analyses, or interpretation of data; in the writing of the manuscript, and in the decision to publish the results.

\section{Abbreviations}

The following abbreviations are used in this manuscript:

PA56: Polyamide 5.6

OSP: Olive stone powder

FTIR: Fourier transform infrared

\section{References}

1. Plastics Europe AISBL. Plastics - the Facts 2019. 2019, 1-42. Available online: https://www.plasticseurope.org/application/files/9715/7129/9584/FINAL_web_version_Plastics the facts2 019 14102019.pdf (accessed on 14 October 2020).

2. Aeschelmann, F.; Carus, M. Bio-based Building Blocks and Polymers in the World: Capacities, Production and Applications-Status Quo and Trends Towards 2020. Industrial Biotechnology 2015, 11, 154-159.

3. Li, M., Li, D., Huang, Y., Liu, M., Wang, H., Tang, Q., \& Lu, F. Improving the Secretion of Cadaverine in Corynebacterium glutamicum by Cadaverine-Lysine Antiporter. J. Ind. Microbiol. Biotechnol. 2014, 41, 701-709.

4. Shah, A.A.; Hasan, F.; Hameed, A.; Ahmed, S. Biological Degradation Of Plastics: A Comprehensive Review. Biotechnol. Adv. 2008, 26, 246-265.

5. Vroman, I., Tighzert, L. Biodegradable polymers. Materials 2009, 2, 307-344.

6. Koutsomitopoulou, A. F., Bénézet, J. C., Bergeret, A., Papanicolaou, G. C. Preparation and Characterization of Olive Pit Powder as a Filler to PLA-matrix Bio-composites. Powder Technology 2014, 255, 10-16.

7. Naghmouchi, I., Mutjé, P., \& Boufi, S. Olive Stones Flour As Reinforcement In Polypropylene Composites: A Step Forward In The Valorization of the Solid Waste From The Olive Oil Industry. Industrial Crops and Products 2015, 72, 183-191.

8. Naghmouchi, I., Mutjé, P., \& Boufi, S. Polyvinyl Chloride Composites Filled With Olive Stone Flour: Mechanical, Thermal, And Water Absorption Properties. J. of Applied Polymer Science 2014, 131, 183-191.

9. Turkish State Meteorological Service. Available online: https://mgm.gov.tr/veridegerlendirme/sicaklikanalizi.aspx?s=a\#sfB (accessed: 17 October 2020).

10. Vert, M., Doi, Y., Hellwich, K. H., Hess, M., Hodge, P., Kubisa, P., Rinaudo, M., \& Schué, F. Terminology For Biorelated Polymers And Applications. Pure and Applied Chemistry 2012, 84, 377-410.

(C) 2020 by the authors; licensee MDPI, Basel, Switzerland. This article is an open access article distributed under the terms and conditions of the Creative Commons by Attribution (CC-BY) license (http://creativecommons.org/licenses/by/4.0/). 